\title{
A Modifiable Agent-Based Software Architecture for Intelligent Virtual Environments for Training
}

\author{
Gonzalo Méndez \\ Computer Science School \\ Technical University of Madrid \\ Campus de Montegancedo s/n \\ 28660 Boadilla del Monte (Madrid), Spain \\ gonzalo@gordini.ls.fi.upm.es
}

\author{
Angélica de Antonio \\ Computer Science School \\ Technical University of Madrid \\ Campus de Montegancedo s/n \\ 28660 Boadilla del Monte (Madrid), Spain \\ angelica@fi.upm.es
}

\begin{abstract}
During the last years, Intelligent Virtual Environments for Training have become a quite popular application of computer science to education. These systems involve very different technologies, ranging from computer graphics to artificial intelligence. However, little attention has been paid to software engineering issues, and most of these systems are developed in an ad-hoc way that does not allow the reuse of their components or an easy modification of the application, even though some authors claim that the use of agents makes systems be more modifiable. We describe two agent-based software architectures that are intended to be easily extended and modified. The first one was designed using an organizational approach recommended by some agent oriented methodologies. The second one is a redesign of the first architecture using more formal principles and methods of software architecture design. A comparison between both architectures highlights the need to use more formal approaches to design this kind of application, specially if we expect to achieve interchangeability of components between different applications.
\end{abstract}

\section{Introduction}

The development of educational Virtual Environments (VEs) has a quite short history, dating from the midnineties. The youth of the field, together with the complexity and variety of the technologies involved, have led to a situation in which neither the architectures nor the development processes have been standardized yet. Therefore, almost every new system is developed from scratch, in an ad-hoc way, with very specific solutions and monolithic architectures, and in many cases forgetting the principles and techniques of the Software Engineering discipline [20].
The MAEVIF project (Model for the Application of Intelligent Virtual Environments to Education) was the result of several experiences integrating VEs and intelligent tutors $[19,18]$ that served to point out the problems that commonly arise in such integrations. The objective of the MAEVIF project was to define a model for the application of intelligent virtual environments to education and training, which involved: the definition of a generic model for intelligent learning environments based on the use of virtual worlds; the definition of an open and flexible agent-based software architecture to support the generic model of an Intelligent Virtual Environment for Training (IVET); the design and implementation of a prototype authoring tool that simplifies the development of IVETs, based on the defined architecture; and the definition of a set of methodological recommendations for the development of IVETs.

Since an IVET is a special kind of Intelligent Tutoring System (ITS), the first approach to define an architecture for IVETs was to use the architecture of an ITS [28] as a starting point (see Figure 1). This architecture, however, does not fully support some features of an IVET, such as multistudent training, immersion in the VE or the interaction required in VEs. Therefore, we extended the ITS architecture by considering additional subsystems, such as a world module, which contains geometrical and semantic information about the 3D representation of the VE and its inhabitants, as well as information about the interaction possibilities.

In the remainder of this paper we describe how this structure has been transformed into an agent-based architecture, and we discuss the features of the resulting system (section 2 ). Then, we show how it has been redesigned and discuss why the resulting architecture is more modifiable than the first one (section 3). After describing both architectures, we show some related work where agents have been used to develop IVETs (section 4). Finally, we present some conclusions and ongoing work (section 5). 


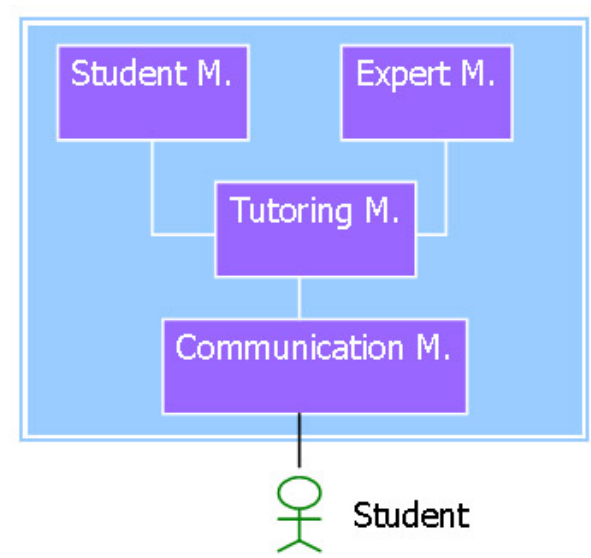

Figure 1. Architecture of an ITS

\section{An Agent-Based Architecture for IVETs}

There are two main reasons why we have chosen agents to develop this system instead of a more traditional approach, either object or component oriented. The first reason, as described in [32] is the fact that, given the increasing complexity that the development of IVETs involves, agents represent a powerful tool to use abstraction as a way to face complexity. In addition, the fact that many agent platforms are developed on top of object oriented languages makes it possible to take advantage of all the possibilities provided by these languages (i.e. JADE and Java).

The second reason is that, although a widely accepted definition of agent does not currently exist, many authors agree on a set of features that agents must have, among which we can find both proactivity and situatedness. Given the fact that IVETs are a highly interactive kind of application, proactivity is a feature that is very well suited for their development, since it makes it easier to design tutors that interact with the students in a human-like way. In addition, training inside an IVET makes it necessary for the tutor to be aware of the structure and state of the environment where that training is taking place. Therefore, situatedness is a feature that makes it possible to manage that information in a more natural way.

This does not mean that the mere use of agents is the solution for all problems, but properly used they are likely to ease the design and implementation of a suitable solution.

\subsection{A Hierarchical Approach}

Taking the structure described in the previous section as a starting point, the next step was to decide which software agents were necessary to transform it into an agentoriented architecture, which has been designed using the
GAIA methodology [32]. In this methodology, the authors suggest the use of the organizational metaphor to design the system architecture, which requires the analysis of the realworld organization in order to emulate its structure. This approach does not always work (depending on particular organization conditions), but in this case, considering the architecture of an ITS as the organization to reproduce, it is possible to imitate its structure to develop the system architecture. There is an additional reason to use an ITS structure as a starting point: the ITS architecture shown in Fig. 1 is widely used by the educational software community. Therefore, if we aim at being able to exchange elements between different applications, making use of a widely used structure is likely to facilitate this task.

Figure 2 shows how the extended ITS architecture was transformed, from a modular point of view, into an agentbased architecture. It has five agents corresponding to the five key modules of the extended ITS architecture: Communication Agent, Student Modelling Agent, World Agent, Expert Agent and Tutoring Agent.

Analyzing the responsibilities of these agents, some additional roles can be identified that point to the creation of new, subordinate agents that can carry them out, subsequently giving rise to a hierarchical multi-agent architecture. Each subordinate agent is in charge of managing some process and the information related to it, while each supervisor agent is in charge of coordinating its subordinate agents and communicating them with other subordinate agents through their respective supervisor.

The Central Communication Agent is responsible for the communication between the VE and the Tutoring System. It delegates part of its responsibilities to a set of Individual Communication Agents dedicated to each student. There is also a Connection Manager Agent, which is responsible for coordinating the connections of the students to the system, and a set of Device Agents in charge of managing the data provided by the devices the students use to interact with the VE.

The Student Modelling Agent is in charge of maintaining a model of each student, including personal information, their actions in training sessions, and a model of the students' knowledge. To do this, the Student Modelling Agent is assisted by: a Historic Agent, which is responsible for registering the history of interactions among the students and the system; a Psychological Agent, which is responsible for building a psychological profile of each student including their learning style, attentiveness, and other personality traits, moods and emotions that may be interesting for adapting the teaching process; a Knowledge Modelling Agent, which is responsible for building a model of the student's current knowledge and its evolution; and a Cognitive Diagnostic Agent, which is responsible for trying to determine the causes of the student's mistakes. 


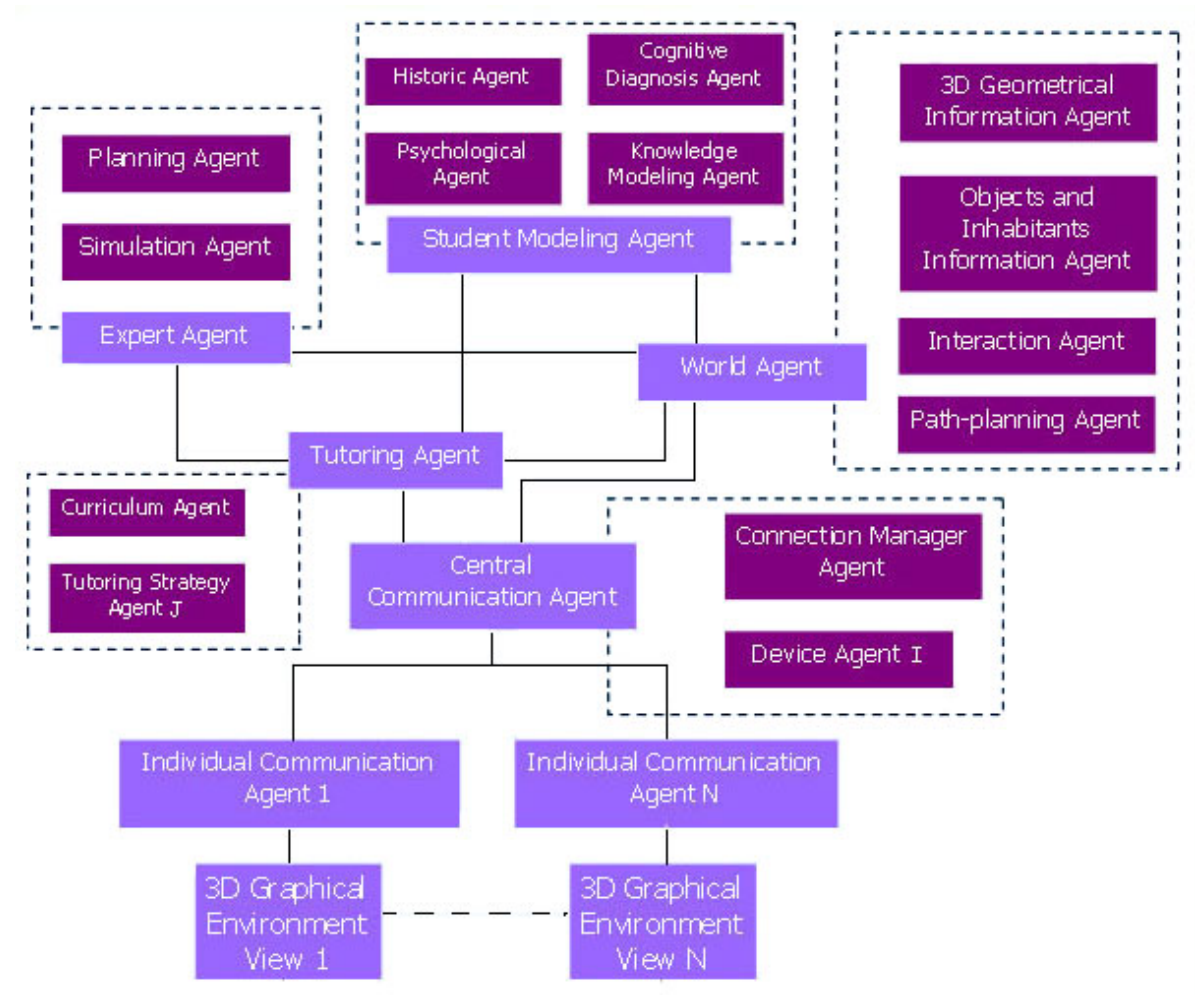

Figure 2. Decomposition view of the agent-based architecture

The World Agent is in charge of maintaining a coherent model of the VE, so that all the agents and students have the same information about the state of the world. It is related to: the 3D Geometrical Information Agent which has geometrical information on the objects and the inhabitants of the world and is able to answer questions about the location of the objects; the Objects and Inhabitants Information Agent, which has semantic knowledge about the objects and the inhabitants of the world and is able to answer questions about the utility of the objects or the objects being carried by a student; the Interaction Agent, which has knowledge about the possible actions that the students can perform in the environment and the effects of these actions and the Path-Planning Agent, which is capable of finding paths to reach a destination point in the environment avoiding collisions with other inhabitants and objects.

The Expert Agent contains the expert knowledge about the environment that is being simulated, as well as the knowledge necessary to solve the problems posed to the student and to reach the desired goals. It delegates some of its responsibilities to a Simulation Agent, that contains the knowledge about the simulated system, and a Planning Agent, that is able to find the best sequence of actions to solve different activities. The plan for an activity is worked out by the Planning Agent with the collaboration of three other agents: the Path-Planning Agent can determine whether there is a trajectory from a certain point of the world to another one; the Interaction Agent provides information about the actions that a student can directly execute in the environment; the Simulation Agent provides information about some high-level actions that can be executed over the simulated system.

The Tutoring Agent is responsible for proposing activities to the students, monitoring their actions in the virtual environment, checking if they are valid or not with respect to the plan worked out by the Expert Agent, and making tutoring decisions. The activities that can be proposed by the Tutoring Agent are dependent on the particular environment that is being simulated in the IVET, and they are defined by means of an authoring tool. The Tutoring Agent is assisted by a Curriculum Agent, which has knowledge of the curricular structure of the subject matter, and several Tutoring Strategy Agents, which implement different tutoring strategies.

The proposed architecture has been implemented using JADE (Java Agent DEvelopment Framework), while the VE has been built using $\mathrm{C}++$ and OpenGL. The communication between the agents and the VE is made using a CORBA middleware, which has allowed us to distribute the different elements of the training application in different machines. 
Some information has to be exchanged between the different VE clients that correspond to each student, such as changes in the positions of the avatars and objects. Microsoft's DirectPlay library has been used with this purpose.

\subsection{Discussion}

All along the design and development of the architecture, one of the aspects that has had a bigger impact on it has been the planning process, since, due to the fact that it is a collaborative task, a change in the planning method or in the way that knowledge is represented may imply changes in all the agents that take part in it. There were two factors that suggested that collaborative planning was a good solution. The first one was the fact that, given a planning algorithm, this solution allows for the real-time inclusion of new agents with different knowledge that can help to solve a problem. In addition, a careful design of the operators and their responsibilities can minimize the impact of a change in the planning algorithm or in the knowledge representation.

At the beginning, a simple STRIPS planner [9] was implemented. However, trying to substitute it with one based on SHOP2 (Simple Hierarchical Ordered Planner 2) [21] showed that it was far more complicated and required more changes than expected. The STRIPS planner provides a very basic planning capability, and more sophisticated functionalities were required, together with an efficient planing method, since real-time performance is quite desirable in IVETs. However, the implementation of such a planner requires a detailed knowledge in the field, so we decided to used an already implemented planner (in addition, this is one of the objectives we are aiming at: the possibility to interchange different components without having to design them to suit our specific needs). The way in which planners are implemented makes it very difficult to add new knowledge interactively. On the contrary, planners usually need to have all the domain defined previous to running the planning algorithm. Therefore, a different approach for planning was required.

Another aspect we tested was how easy it was to add new functionality to the IVET. To do this, we added an embodied tutor whose goal was to observe what happened in the VE and follow the student to supervise him. It was necessary to add two new agents, namely the Virtual Tutor agent, whose responsibility was to control the $3 \mathrm{D}$ representation of the tutor (its embodiment), and the Perception agent, who was in charge of monitoring the events of the virtual world. Both of them were under the supervision of the World agent. Although it was quite easy to make these changes, it soon became clear that any non-trivial change was likely to affect at least one of the supervisor agents (the one that supervises the modified agent) if not more. In addition, all the agents knew of the existence and identity of the agents they had to communicate with, so they were easily affected by changes.

There were some other factors that made us think that a redesign of the architecture was necessary, both at design and implementation levels. One of them was the poor performance the system offered when several students were taking part in a training session. Several tests pointed out that the agent platform presented a fairly good performance, and so did the VE. The problem arose when running both of them together, although in different machines, which made us think of poor communication performance. In addition, we were having problems when trying to add new functionality, since it was not clear whether some responsibilities were to be assigned to the expert agent or to the world agent, both of which started to be too coupled for the system to be modifiable. This is a problem that usually arises when establishing classifications and hierarchies: if the criteria used for classification changes or some elements don't fully fall under one of the categories, then the decomposition degrades quite quickly. This was the case with our architecture.

Finally, there were other facts that pointed out the unsuitability of the architecture. Among them, some are described in [31] as an indication of a poor design, such as the proliferation of agents to carry out small tasks, the difficulty to assign responsibilities to an agent or the existence of agents that carry out actions for which an agent is not needed.

These problems were caused, at least partially, by the lack of an architectural design method in a not very mature field as multi-agent systems are.

\section{Redesigning the Architecture}

After analyzing the results obtained with the organizational approach, we decided to redesign the architecture making use of not specifically agent oriented software engineering techniques. The redesign had the objective of obtaining an easily modifiable architecture, that allowed us to substitute virtually any agent by a different one with a low impact on the other agents. In addition, we also wanted to be able to extend the system with as few modifications as possible.

The main theoretical support to redesign the architecture has been the body of work on software architecture developed at the Software Engineering Institute (SEI) [2, 6, 7], such as Attribute Driven Design (ADD), Architecture Tradeoff Analysis Method (ATAM) and Views and Beyond (V\&B). The purpose of the first two methods is to design and evaluate a software architecture driven by the quality attributes desired for it, instead of only the functionality. A set of scenarios is used to help identify the quality attributes that are relevant for the architecture, based on the stakeholders interests, and to evaluate the architecture in order to identify potential risks. 
The other important support has been provided by the use of information hiding [23], which establishes that a division in submodules must be such that each submodule encapsulates a design decision that must remain hidden from the rest, and communication among submodules is carried out using an interface with a definition as abstract as possible. The design decisions that are encapsulated in each module are related to the changes that are perceived to be likely over the system's life. The way to proceed, then, is to use abstraction as a means to face complexity and facilitate changes.

Although we planned to use ADD as the architectural design method, we discarded it after a few design sessions because of two reasons. The first reason is the fact that ADD is based on a hierarchical system decomposition, and one that does not allow elements to have more than one father. After the experience gained with the previous architecture, we did not think a hierarchical structure was what we wanted to obtain. In addition, some of the problems we had with the first architecture was the fact that some agents were not clearly under the supervision of one of the five supervisor agents. The second reason has to do with the complexity of decomposing a module in more than four or five elements, which was likely to be the case (in the current design, the ITS consists of nine different kinds of agents).

We soon found two more grounded reasons to discard ADD as a design method. The first one is Parnas argument expressed in [24], where he clearly states that, although maybe desirable, information hiding and hierarchical structure do not always go together. On the contrary, we consider information hiding to be a design criterium, and not just a decomposition one. The other reason can be found in [1], where the author analyzes Simon's "Architecture of Complexity" and identifies the historical reasons that made hierarchical structure a predominant design mechanism.

Agent systems are intrinsically peer-to-peer (after all, they were born in the distributed artificial intelligence field), where each agent is a peer that makes use of services offered by other agents to carry out the responsibilities assigned to it. Therefore, this is the approach we have followed to design the new architecture. Like ADD suggests, we have started by selecting the architectural drivers for our application. However, instead of following a decomposition approach, we have worked using an iterative an incremental approach. A sketch of the architecture was always present in a whiteboard in the architectural design sessions. Every time a feature had to be added, or a change had to be made, it was tested against the architectural drivers until a way was found to satisfy them. In that moment, the change was added to the architectural design.

This is probably one of the issues ADD still has to address, since it is not always possible to face a new design or modification as a hierarchical decomposition.

\subsection{Quality Attributes}

The design started with the definition of a set of quality scenarios to establish what kind of changes were to be considered by the design. In general, this changes have to do with the ability to substitute an agent with a different one that provides a similar functionality, or to move some responsibility from one agent to another. This is required because one of the objectives of the system is to be used as a test-bed for teams developing just some of the elements of the ITS (e.g. the student modelling or the tutoring strategy).

Another kind of change is the possibility to turn off some functionality, such as supervision, so that the student can use the system in an exploratory way without the tutor interrupting him (although the system would continue registering his actions), or even disabling tutoring completely.

The system is also required to be easily extended, so that new agents that provide new functionality can be added without having to make changes in the existing ones (at least, in the ones that don't make direct use of the new functionality).

Taking into account that training is carried out in a VE, all these modifiability requirements cannot be an obstacle for the main objective of the system, which is to provide students with a training environment as similar as possible to the real one. For that, it is of utmost importance to keep performance close to real time. If not, the training experience may be somehow frustrating for the student, which may cause the training experience to be less efficient than other, more traditional, methods.

There is a usability attribute, adaptation to the user, that has not been considered explicitly because it is already included in the objectives of an ITS. The student modelling is used to personalize the training process to the student's abilities and needs, so it has not been necessary to consider it as an additional quality attribute to take into account.

\subsection{Design Decisions}

With the described modifiability objectives in mind, the approach we followed was to keep the agents as anonymous as possible, so that no agent directly knows what other agents are carrying out the actions they need to successfully complete their responsibilities. To achieve this, during system startup, the agents announce in the system's yellow pages the services they are capable to provide to other agents. Thus, an agent does not know how many or what kind of agents there are in the system; they just know that there is an agent that can provide a certain service they need. This way, it is easier to change the agent that provides a service, as long as the service is provided in the same terms the original one was. This requirement is similar to Liskov's substitution principle in object oriented design [16]. 
Once the agent finds the service it is looking for, it can act in two different ways. If the service involves frequent updates, the agent subscribes to an update list, so that every time an update arrives, it is immediately informed about it. If, on the contrary, the agent only needs to request the service at specific times, it annotates which agent it has to request the service to. In both cases, the decision is made at runtime, so changes in the design are easier to carry out. We considered the possibility of giving the chance to change the service provider any time during runtime, but we discarded it because it is not likely to happen in a system of this kind.

The agents communicate with each other exchanging FIPA ACL messages. Since it is a quite extended formalism, the difficulties may come from the communication protocol. We have designed a fairly simple communication protocol for a given agent to request a service from another agent. Agent A sends a request to agent B, who acknowledges the reception of the request. Then agent $B$ carries out the required actions and sends agent $\mathrm{A}$ the result of the execution of the service (or a message with the reasons why it could not be carried out). Agent A acknowledges the reception of the result and the communication stops until another service request is required.

A similar mechanism has been used to communicate the agent platform with the VE, but with an even simpler communication mechanism. A communication centre has been designed where both the agent platform and the VE send their messages for other applications to receive them. Each application subscribes to the messages it is interested in receiving, so that, for example, different VEs or different versions of a VE only receive the messages they know how to handle. We have used this possibility all along the development of the agent platform, so we could send the messages we wanted to test from a console instead of having to run the VE and carry out a specific procedure before the message we were interested in could be sent.

We have also made use of configuration files to set up the training session. Thus, the description of the procedures to be trained, the composition of the scenarios, the objectives of the activity, its participants or the parametrization of the tutoring strategy are all read from several configuration files, which allows changes in the way the system behaves without further changes in the design.

\subsection{Resulting Architecture}

The resulting architecture is the one shown in Fig. 3. The picture shows the structure of the architecture as it is currently designed, where all the agents are represented along with the communication channels (the yellow pages are not represented, since all the agents communicate with them).

During runtime, there is only one agent of each kind, except for the agents that are directly related with students: the
Student Modelling Agent and the Communication Agent. This is so because, having several students, the system can handle the communication with them in parallel. In addition, if the agent platform needs to be distributed in different machines, the distribution can be made in terms of the number of students.

The main differences with the former architecture are:

- There is no hierarchical structure. Since the existence of the supervisor agents was due to modifiability reasons, and it was only achieved in a low degree, it has been considered preferable to use a peer-to-peer style.

- It uses a publish-subscribe style to offer a service oriented behaviour. Agents advertise their services in the yellow pages and other agents can subscribe to the services they are interested in. Although we were not aiming at obtaining a Service Oriented Architecture, this is one of the mechanisms that introduces a higher degree of modifiability in the system.

- Task planning is not a collaborative task any more. The planning agent acts as a wrapper [11] for the planner, hiding details of its functioning to the rest of the system and enabling the change of the planing algorithm with a lower impact than collaborative planning had.

- Extended support for a simulator. Some systems such as the one described in [17] simulate environmental events that may be caused by external factors, such as changes in the state of a patient. In the cited system, events are directly simulated in the VE, but it may also be desirable to use an external simulator in cases where it has already been implemented or when it has a complex behaviour. The simulation agent can now simulate simple systems, but it can also receive information from a simulation running together with the VE or act as a wrapper of an external simulation.

- The tutoring strategy can be adjusted by changing some parameters that are read from a configuration file during the initialization of the system. These parameters are expected to change dynamically with the new design of the student modelling agent.

- The world agent is responsible for maintaining an ontology that stores the state of the VE. A simple reasoning engine has been added so that the world agent is able to provide richer answers to the student.

- A message centre is now used to communicate the different subsystems that form the training system. Each subsystem registers in the message centre and requests the kind of information it is interested in. Currently, in addition to the VEs and the ITS, a command line console can also connect to the message centre with debug purposes. 


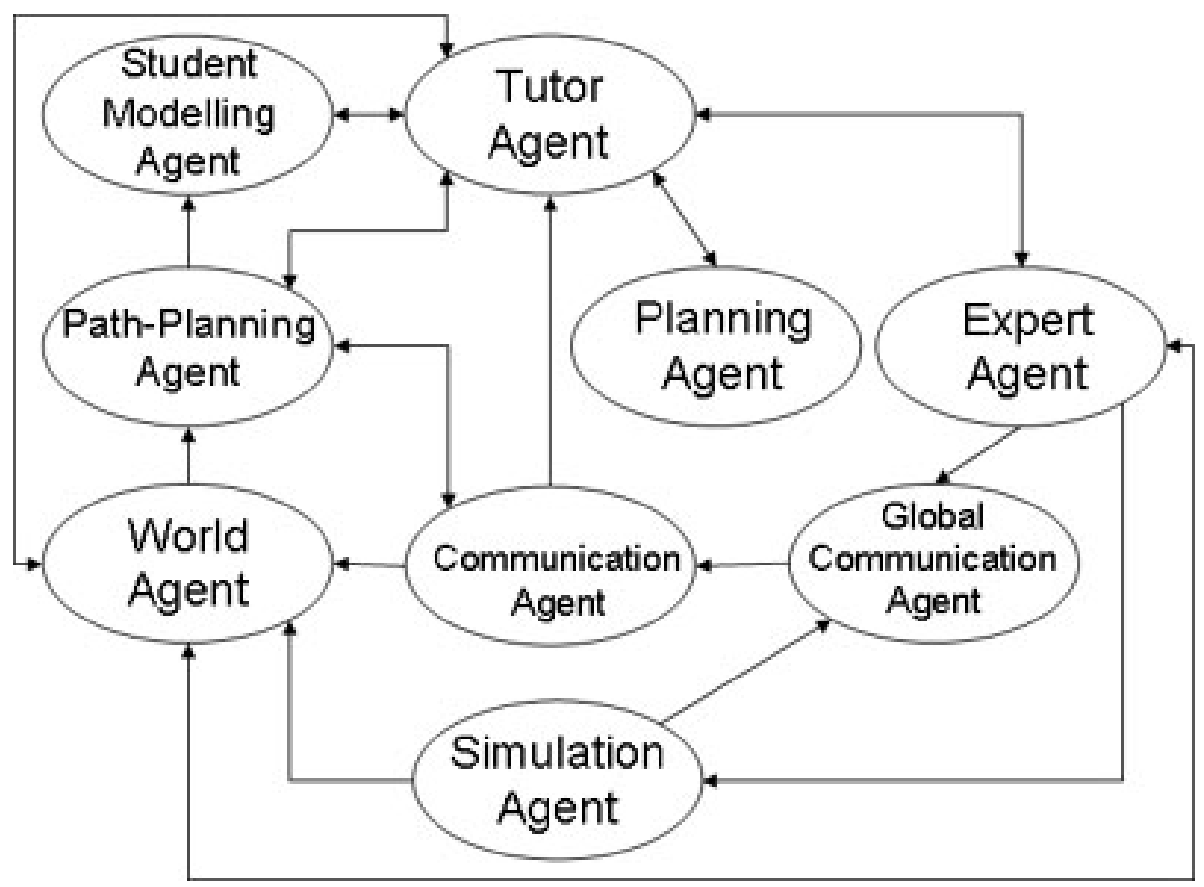

Figure 3. Peer-to-peer view of the new architecture. Ellipses represent agents and arrows show communication channels

\subsection{Evaluation}

In addition to the more formal design and documentation of the software architecture, we are currently evaluating the suitability of the architecture to our needs both at architectural and runtime levels.

At the architectural level, the evaluation requires the use of quality scenarios provided by the stakeholders to identify relevant quality attributes We are trying to gather a thorough collection of scenarios that gives us a better understanding of the implications of the design decisions we have made. In order to do it, we have run an ATAM session and we are planing to run another one in the context of a 3-year research project, ENVIRA, that has already started in conjunction with two other research groups that will be using the multiagent system to develop their own training systems.

In the first session, the participants were the members of the development team, and we only made use of some steps of Phase 0 and of Phase 2 of ATAM, as described in [7], since all of them were familiar with the architecture. The main objectives of this session were to identify and evaluate use case scenarios and growth scenarios.

Given the composition of the group that took part in the evaluation, no significant results were obtained in terms of use case scenarios, although we have been able to check that the design decisions we made while designing the architec- ture were still valid. As for the growth scenarios, we were able to identify sensitivity points that we will have to cope with in the ENVIRA project. These sensitivity points have to do with the addition of a new student modelling scheme and a cognitive architecture for virtual characters managed by agents.

We have already scheduled a second ATAM session where the members of the other two research teams will also take part. In this session, we expect to get more results about growth scenarios related to their assignments in the project and a few exploratory scenarios provided by the members of the three groups that are taking part in the ENVIRA project.

There is not a complete report on the results of the evaluation of the architecture yet, but we intend to have it as soon as the second ATAM workshop is finished.

To test the system at runtime level, we are developing it in an iterative way. Each agent is being developed apart from the rest of the system, and the agents they need to communicate with have been substituted by 'dummy' agents. At the end of each iteration, the dummy agents are removed and substituted by the agents that are under development. This way, the development keeps focused on three aspects: adherence to the designed communication protocols; change of one agent by a different one, even if it is as simple as the dummy agents are; turning some functionalities on and off, with the aid of the dummy agents (although 
another mechanism is to be designed so that the dummy agents are not necessary to turn off functionalities).

There is already a functional pilot application that offers much of the functionality that the previous version provided. For the moment, the student modelling is quite simple, as well as the simulation agent. In contrast, the tutoring agent is capable of supervising the student, providing different levels of hints and answers to the student's questions. The planning agent is already capable of planning a procedure and replanning alternatives in response to the student's actions, and the world an expert agents provide support to the tutoring agent, so that it can provide better assistance to the student according to the state of the environment and the characteristics of the procedure the student is training. Both the agent platform and the VE show an adequate performance when running at the same time in the same or different machines, either with one or two students. Further testing is needed to add more students, but with the current results we expect the system to behave better than the previous version.

\section{Related Work}

There are several projects aiming at the use of VR for education and training supported by intelligent agents. The first ones were developed over a decade ago, and the most representative among them are Steve [25], Adele [26], Cosmo [15], Herman the Bug [14] and Vincent [22]. What all of them have in common is the fact that the primary objective in all of them was to develop an embodied pedagogical agent to support education and training. Each of them tried to solve some of the problems that this emerging discipline posed.

None of these systems are structured as multiagent systems, but as a single agent that inhabits a particular virtual world, and each of them exhibits its own internal architecture. Even so, they have been the key to identify some of the issues that researches are still trying to solve in a satisfactory way.

There are some examples of multiagent systems that support education and training without using VEs. That is the case of FILIP, a multiagent system for training based on simulations [33] to provide training for air controllers. The system is composed by seven agents that cover the modules of an ITS: one for the student, one for the expert, three for the tutor (skill development, curriculum and instructor agents) and two other agents related to the communication with the learning environment and the user.

Baghera is another example of multiagent system used to teach geometry [27]. The aim of this system is to study emergent behaviours in multiagent systems. What makes this system more interesting is the fact that agents are organized in two levels, and the number of agents is not fix, but varies according to the number of students connected to the system. Each student is assisted by three agents: the personal interface agent, which monitors the student's actions, the tutor agent and the mediator agent. In addition, the tutor is assisted by two agents: the personal interface agent and the assistant agent. All these agents are supported by second level agents of four different kinds, which are in charge of evaluating the student's actions. This is made through a voting mechanism that causes the emerging behaviours that are the subject of study.

The systems that are closer to the one described in this paper are those that are based on multi-agent systems and make use of VEs to support training. A good example is MASCARET (Multi-Agent Systems to simulate Collaborative, Adaptive and Realistic Environments for Training) [4], an agent-based IVET that has been used to train firemen in operation management. In this system, agents are divided in organizations, each of which controls different aspects of the organization: physical, social, pedagogical, mediation, and human interaction. The agents that integrate the pedagogical organization cover the four modules of an ITS, plus a fifth module that is in charge of controlling the mistakes an student may make. The expert agent communicates with the social and physical organizations to be able to know what to to and what objects and agents are involved in an action.

Lahystotrain is an IVET developed to train surgeons in laparoscopy and hysteroscopy interventions [17]. This systems contains five agents which help the student in the training process. One of them is the tutor, which supervises the student and registers his actions. An assistant agent provides explanations and interrupts him when he makes a mistake. These agents have an ad-hoc architecture tailored to suit their responsibilities. The other three agents take the role of an auxiliary surgeon, a nurse and an anaesthetist that play their role within the team. Their architecture is the same for all three, and it is a kind of BDI architecture with a perception module, a reasoning engine and an action control module. The student must learn what the role of these three agents is and how to coordinate them.

What most of these systems have in common is the fact that they have been developed to solve a specific problem, but only a few of them have been designed to be reusable, at least to some extent $[25,19,8]$, and apparently none of them have taken advantage of the existing knowledge on software architecture. This is an aspect that was already put forward in [5], where three main problems are mentioned: the fact that most research groups develop only part of the systems, which does not give them a view about the whole design; systems are tailored to solve specific problems; and designs are not evolutive. They claim that an ITS can be developed as a set of independent agents that exchange messages in a predefined language, and they use the concept of federated architecture [10] to articulate their system GIA. 
To some extent, the architecture described in this paper follows those guidelines, although the federated architecture has been substituted by a service oriented approach and a more formal software engineering support has been used.

There are a few examples of multiagent systems that have been evaluated using ATAM. None of them report the use of a specific method to design the architecture, but the fact that they use ATAM is an evidence that the agent community is starting to recognize the need to pay more attention to architectural design.

Among these systems, we can highlight the work presented in [3], where the authors report a successful utilization of an ATAM workshop to evaluate an agent-based software architecture for an industrial transportation system. Although they do not explain the method that was used to design the architecture, they suggest they took quality attributes into account when designing it.

Another work reporting the use of ATAM is the one described in [30], which is the first reference we have found about the use of ATAM to evaluate a multiagent system. In this work, the authors put forward what they think to be relevant attributes in agent-based systems: performance predictability, security against data corruption and spoofing, resilience to modifiability of the environment and availability and fault tolerance. Although not all of them are applicable to the system presented in this paper, this work constitutes an important approach from agent-oriented software development to more traditional software construction.

\section{Conclusions and Ongoing Work}

It is getting common for Virtual Environments for Training to be designed as Multi-Agent Systems, since agents provide a higher level of abstraction than objects and this helps to face the increasing complexity that involves the development of these systems.

Many authors claim, without further proof, that their systems are flexible because they are using agents to build them, and to some extent we may have made the same mistake in our first version of the architecture. Although it had been designed with modifiability in mind, it soon became clear that successive modifications were making the architecture degrade quite quickly. That experience is one more example to show that the mere use of agents (or any other technology) does not guarantee that the application developed using them will have certain properties. On the contrary, the result may be even worse if the design decisions have not been made with care.

In the second version of the architecture, we have tried to take advantage of the growing experience in the field of software architecture, even if it is not specifically agent oriented (something that is not considered to be necessary at the architectural level [2]). As with the previous version of the architecture, only time and changes will prove whether we made the right design decisions or not, but the fact that this time the architecture was more carefully crafted and the current results make us think so.

However, we have not been able to use ADD for the architectural design, given the fact that a hierarchical decomposition does not seem to suit our needs. A review of the new version of ADD [29] shows it is based on the same design strategy, so we still need a different design approach that is not based on hierarchical structure and decomposition. Even so, designing with quality attributes as architectural drivers as ADD promotes has resulted in a design that, up to now, has proven to be more modifiable than the previous design was.

As for the use of ATAM, it is a valuable tool from which we still expect to obtain useful results as soon as the second workshop is carried out

We are already making changes to the system to test to what extent it can be modified, and they are being evaluated both on the architectural design and on the implemented system. In addition to the modification of the student modelling agent, there are two main changes that will prove the suitability of the architecture. The first one is the inclusion of a model of human-like perception [12] to use the student's attention as part of the student's model and as an additional source of information for tutoring decisions. The second one is the inclusion of a cognitive architecture that allows us to make use of virtual tutors and teammates with complex, emotional behaviours [13].

\section{Acknowledgements}

This research has been funded by the Spanish Ministry of Science through projects MAEVIF (TIC2000-1346), ICEVAPI (TIN2004-07946) and ENVIRA (TIN2006-15202C03-01) and has been supported by the INTUITION NoE.

\section{References}

[1] P. E. Agre. Hierarchy and history in simon's "architecture of complexity". Journal of the Learning Sciences, 12(3):413426, 2003.

[2] L. Bass, P. Clements, and R. Kazman. Software Architecture in Practice. SEI Series in Software Engineering. Addison Wesley Professional, 2nd edition, 2003.

[3] N. Boucke, D. Weyns, K. Schelfthout, and T. Holvoet. Applying the atam to an architecture for decentralized control of a transportation system. In C. Hofmeister, I. Crnkovic, R. Reussner, and S. Becker, editors, 2nd International Conference on the Quality of Software Architectures (QoSA). Springer-Verlag, 2006.

[4] C. Buche, R. Querrec, P. D. Loor, and P. Chevaillier. Mascaret: A pedagogical multi-agent system for virtual environments for training. International Journal of Distance Education Technologies, 2(4):41-61, 2004. 
[5] B. Cheikes. Gia: An agent-based architecture for intelligent tutoring systems. In T. Finin and J. Mayfield, editors, Proceedings of the CIKM'95 Workshop on Intelligent Information Agents, Baltimore, Maryland, 1995.

[6] P. Clements, F. Bachmann, L. Bass, D. Garlan, J. Ivers, R. Little, R. Nord, and J. Stafford. Documenting Software Architectures: Views and Beyond. The SEI Series in Software Engineering. Addison Wesley Professional, 1 edition, 2002.

[7] P. Clements, R. Kazman, and M. Klein. Evaluating Software Architectures. The SEI Series in Software Engineering. Addison Wesley Professional, 1 edition, 2002.

[8] M. Evers and A. Nijholt. Jacob - an animated instruction agent in virtual reality. In T. Tan, Y. Shi, and W. Gao, editors, Advances in Multimodal Interfaces - ICMI 2000, Proceedings of the Third International Conference on Multimodal Interfaces, number 1948 in LNCS, pages 526-533. Springer-Verlag, 2000.

[9] R. E. Fikes and N. J. Nilsson. Strips: a new approach to the application of theorem proving to problem solving. Artificial Intelligence, 2(3-4):189-208, 1971.

[10] M. Genesereth. An agent-based approach to software interoperability. Technical Report Logic916, Logic Group, Computer Science Department, Stanford University, 1993.

[11] S. Hayden, C. Carrick, and Q. Yang. Architectural design patterns for multi-agent coordination. In Proc. of the 3rd Intl. Conf. on Agent Systems (Agents'99), 1999.

[12] P. Herrero and A. de Antonio. Keeping watch: Intelligent virtual agents reflecting human-like perception in cooperative information systems. In Proc. of the 11th Intl. Conf. on Cooperative Information Systems. Springer-Verlag, 2003.

[13] R. Imbert and A. de Antonio. Using progressive adaptability against the complexity of modeling emotionally influenced virtual agents. In Proc. of the 18th Intl. Conf. on Computer Animation and Social Agents (CASA 2005), 2005.

[14] J. Lester, B. Stone, and G. Stelling. Lifelike pedagogical agents for mixed-initiative problem solving in constructivist learning environments. User Modeling and User-Adapted Interaction, 9(1-2):1-44, 1999.

[15] J. Lester, J. Voerman, S. Towns, and C. Callaway. Cosmo: A life-like animated pedagogical agent with deictic believability. In IJCAI97 Workshop on Animated Interface Agents: Making them Intelligent, Nagoya, Japan, August 1997.

[16] B. Liskov and J. Wing. Family values: A behavioral notion of subtyping. Technical Report MIT/LCS/TR-562b, Cambridge, MA, USA, 1993.

[17] J. L. los Arcos, W. Muller, O. Fuente, L. Ore, E. Arroyo, I. Leaznibarrutia, and J. Santander. Lahystotrain: Integration of virtual environments and its for surgery training. In G. Gauthier, C. Frasson, and K. VanLehn, editors, Intelligent Tutoring Systems (2000), number 1839 in LNCS, pages 43-52. Springer-Verlag, 2000.

[18] G. Mendez, P. Herrero, and A. de Antonio. Intelligent virtual environments for training in nuclear power plants. In Proc. of the 6th Intl. Conf. on Enterprise Information Systems (ICEIS 2004), Porto, Portugal, April 2004.

[19] G. Mendez, J. Rickel, and A. de Antonio. Steve meets jack: the integration of an intelligent tutor and a virtual environment with planning capabilities. In 4th Intl. Working Conf. on Intelligent Virtual Agents (IVA03), volume 2792 of LNAI, pages 325-332, Kloster Irsee, Germany, September 2003. Springer-Verlag.

[20] A. Munro, D. Surmon, M. Johnson, Q. Pizzini, and J. Walker. An open architecture for simulation-centered tutors. In Proc. of AIED99: 9th Conference on Artificial Intelligence in Education, pages 360-67, Le Mans, France, 1999.

[21] D. Nau, T. Au, O. Ilghami, U. Kuter, W. Murdock, D. Wu, and F.Yaman. Shop2: An htn planning system. Journal of Artificial Intelligence Research (JAIR), 20:379-404, 2003.

[22] A. Paiva and I. Machado. Life-long training with vincent, a web-based pedagogical agent. International Journal of Continuing Engineering Education and Life-Long Learning, 12(1), 2002.

[23] D. L. Parnas. On the criteria to be used in decomposing systems into modules. Communications of the ACM, 15(12): 1053 - 1058, December 1972.

[24] D. L. Parnas. On a 'buzzword': Hierarchical structure. In Information Processing 74, Proceedings of IFIP Congress 74, pages 336-339, 1974.

[25] J. Rickel and W. L. Johnson. Animated agents for procedural training in virtual reality: Perception, cognition, and motor control. Applied Artificial Intelligence, 13:343-382, 1999.

[26] E. Shaw, W. Johnson, and R. Ganeshan. Pedagogical agents on the web. In Proceedings of the Third Annual Conference on Autonomous Agents, pages 283-290, Seattle, WA, USA, May 1999. ACM Press.

[27] C. Webber and S. Pesty. A two-level multi-agent architecture for a distance learning environment. In E. de Barros Costa, editor, Workshop on Architectures and Methodologies for Building Agent-based Learning Environments (ITS 2002), pages 26-38, 2002.

[28] E. Wenger. Artificial Intelligence and Tutoring Systems. Computational and Cognitive Approaches to the Communication of Knowledge. Morgan Kaufmann Publishers, Los Altos, California, 1987.

[29] R. Wojcik, F. Bachmann, L. Bass, P. Clements, P. Merson, R. Nord, and B. Wood. Attribute-driven design (add), version 2.0. Technical Report CMU/SEI-2006-TR-023, CMU/SEI, 2006.

[30] S. G. Woods and M. Barbacci. Architectural evaluation of collaborative agent-based systems. Technical Report CMU/SEI-99-TR-025, CMU/SEI, 1999.

[31] M. Wooldridge and N. R. Jennings. Software engineering with agents: Pitfalls and pratfalls. IEEE Internet Computing, 3(3):20-27, May-June 1999.

[32] F. Zambonelli, N. R. Jennings, and M. Wooldridge. Developing multiagent systems: The gaia methodology. ACM Transactions on Software Engineering and Methodology (TOSEM), 12(3):317-370, July 2003.

[33] D. Zhang, L. Alem, and K. Yacef. Using multi-agent approach for the design of an intelligent learning environment. In C. Z. Wayne Wobcke, Maurice Pagnucco, editor, Proceedings of the Workshops on Commonsense Reasoning, Intelligent Agents, and Distributed Artificial Intelligence: Agents and Multi-Agent Systems Formalisms, Methodologies, and Applications, number 1441 in LNCS, pages 221230. Springer-Verlag, 1998. 